\title{
An insertion mutation in $A B C B 4$ is associated with gallbladder mucocele formation in dogs
}

\author{
Katrina L Mealey*1,4, Jonathan D Minch'1, Stephen N White2,3,4, Kevin R Snekvik³ and John S Mattoon
}

\begin{abstract}
Background: $A B C B 4$ functions as a phosphatidylcholine translocater, flipping phosphatidylcholine across hepatocyte canalicular membranes into biliary canaliculi. In people, ABCB4 gene mutations are associated with several disease syndromes including intrahepatic cholestasis of pregnancy, progressive familial intrahepatic cholestasis (type 3), primary biliary cirrhosis, and cholelithiasis. Hepatobiliary disease, specifically gallbladder mucocele formation, has been recognized with increased frequency in dogs during the past decade. Because Shetland Sheepdogs are considered to be predisposed to gallbladder mucoceles, we initially investigated $A B C B 4$ as a candidate gene for gallbladder mucocele formation in that breed, but included affected dogs of other breeds as well.
\end{abstract}

Results: An insertion (G) mutation in exon 12 of canine ABCB4 (ABCB4 1583_1584G) was found to be significantly associated with hepatobiliary disease in Shetland Sheepdogs specifically $(P<0.0001)$ as well as other breeds $(P<$ 0.0006). ABCB4 1583_1584G results in a frame shift generating four stop codons that prematurely terminate ABCB4 protein synthesis within exon 12, abolishing over half of the protein including critical ATP and a putative substrate binding site.

Conclusions: The finding of a significant association of ABCB4 1583_1584G with gallbladder mucoceles in dogs suggests that this phospholipid flippase may play a role in the pathophysiology of this disorder. Affected dogs may provide a useful model for identifying novel treatment strategies for ABCB4-associated hepatobiliary disease in people.

\section{Background}

Bile is produced by the collective actions of a number of transporters located on the canalicular membrane of hepatocytes [1]. Active transport of biliary solutes creates an osmotic force that attracts water through tight junctions and aquaporins in the hepatocyte membrane $[2,3]$. Bile salts are the most important biliary solute. Other important solutes of bile include cholesterol and phospholipids. The presence of phospholipids, phosphatidylcholine (PC) in particular, in the biliary lumen is crucial for protecting the epithelial cell membranes lining the biliary system from the cytotoxic detergent actions of bile salts [3-5]. Bile salt cytotoxicity is substantially reduced in the presence of PC owing to the formation of mixed micelles (PC + bile salts) rather than simple micelles (bile salts only). Thus, a decrease in the amount of biliary PC

* Correspondence: kmealey@vetmed.wsu.edu

${ }^{1}$ Department of Veterinary Clinical Sciences, College of Veterinary Medicine, Washington State University, Pullman, WA 99164-6610, USA

Full list of author information is available at the end of the article leads to injury of epithelial cells lining the biliary system [6].

$\mathrm{ABCB} 4$ functions exclusively as a phospholipid translocator [6]. ABCB4 is expressed on cannalicular membranes of hepatocytes where it translocates PC from the hepatocyte to the biliary canalicular lumen [7]. Proper function of $\mathrm{ABCB} 4$ is critical for maintaining hepatobiliary homeostasis as evidenced by the myriad of diseases that occur when polymorphisms of $\mathrm{ABCB} 4$ cause complete or partial protein dysfunction. ABCB4 deficiency is associated with a variety of hepatobiliary disorders in people including progressive familial intrahepatic cholestasis (PFIC type 3 ), cholelithiasis, and cholestasis of pregnancy [4,8-10]. Abcb4-/- mice, in which $A b c b 4$ function is lacking entirely, also develop severe hepatobiliary disease that starts at a few weeks of age and progresses throughout life [11,12].

Hepatobiliary disease in dogs has been recognized with increased frequency during the past several years. In particular, gallbladder mucoceles (mucinous hyperplasia or mucinous cholecystitis) have been documented to be an 
increasingly important cause of hepatobiliary disease in dogs [13-15]. Histopathologic findings associated with ABCB4 associated diseases in people, including intrahepatic cholestasis, cholecystitis, and periportal inflammation $[13,16,17]$, are not commonly reported in dogs with gall bladder mucoceles. Additionally, gallbladder mucoceles are not a component of ABCB4 linked syndromes in people or mice. Gallbladder mucoceles, which occur rarely in people, are often associated with extrahepatic bile duct obstruction. The etiology of gallbladder mucoceles in dogs has not yet been identified, but extrehepatic bile duct obstruction is not commonly associated with this disorder $[14,15]$. Gallbladder mucoceles may result from chronic injury to the epithelial lining of the biliary system since hypersecretion of mucin is the typical physiologic response of any epithelial lining to injury.

Recently Shetland Sheepdogs were identified as a breed that is predisposed to gallbladder mucocele formation, suggesting a genetic predisposition [13]. Because ABCB4 dysfunction is associated with hepatobiliary disease in people and mice, we postulated that a defect in canine $A B C B 4$ might be responsible for gallbladder mucocele disease in dogs, and Shetland Sheepdogs in particular. Therefore, we sequenced canine $A B C B 4$ in affected and unaffected Shetland Sheepdogs as well as affected and unaffected dogs of other breeds.

\section{Methods}

Collection of DNA from affected and unaffected individuals All work was approved by the institutional Animal Care and Use Committee. Collection of DNA from affected Shetland Sheepdogs was accomplished by soliciting owners' cooperation. In order to cast a wide net, owners of dogs with confirmed (ultrasound, surgery, or histopathology) or suspected (elevated liver enzymes - alkaline phosphatase, alanine aminotransferase and/or gamma glutamyl transferase -, total bilirubin, cholesterol and/or triglycerides) gallbladder disease were asked to submit a cheek swab, copy of the dog's pedigree, and copy of the dog's medical record. Contact of Shetland Sheepdog owners was made through the American Shetland Sheepdog Association. For collection of unaffected Shetland Sheepdogs, an additional request for DNA from healthy Shetland Sheepdogs (with confirmatory medical records) was made. For collection of DNA from affected dogs of any breed, records from the Washington Animal Disease Diagnostic Laboratory were searched for canine patients with histopathologic confirmation of gallbladder mucocele. For collection of DNA from unaffected dogs of any breed, a specific solicitation through the Washington State University College of Veterinary Medicine was made for healthy dogs (no history of gallbladder disease) over 9 years of age. In order to increase our confidence in designating a dog as "unaffected", we recruited dogs
(Shetland Sheepdogs and other breeds) greater than 9 years of age. While this may have limited the number of dogs included in the study, it more accurately reflected a dog's true phenotype (affected $v s$. unaffected). A dog was considered 'affected' if a gallbladder mucocele was diagnosed using previously established criteria[13], which included at least one of the following (in order of increasing stringency); ultrasound report by a boarded veterinary radiologist $(\mathrm{n}=3)$, surgical report $(\mathrm{n}=5)$, or histopathologic report $(n=7)$. Dogs with no evidence of gallbladder disease as determined by a normal serum chemistry panel and no apparent physical examination abnormalities were considered 'unaffected'.

\section{Sequencing of canine $A B C B 4$}

Exons 1 through 26 of canine $A B C B 4$ were sequenced after PCR amplification of genomic DNA from affected and unaffected Shetland Sheepdogs. Table 1 contains the sequences of the oligonucleotide primers. Purified PCR amplicons were sequenced with an Applied Biosystems ABI 3730 sequencer (Foster City, CA). Affected and unaffected dogs of other breeds (non-Shetland Sheepdogs) were sequenced only at exon 12 . DNA from all dogs except the 3 affected non-Shetland Sheepdogs was extracted from cheek swab samples. Formalin-fixed, paraffin embedded liver tissue was used for extraction of DNA from these 3 dogs. Samples were processed first using the RiboPure RNA extraction kit (Ambion, Foster City, CA) until step C3. The interphase from this step (containing DNA and protein) was then subjected to DNA extraction using the DNeasy Blood and Tissue Kit (Qiagen, Alameda, CA).

\section{Allele specific PCR}

In order to confirm the insertion mutation in exon 12 (ABCB4 1583_1584G), allele specific primers were designed (mutant: forward 5'- CCTGGTTCGCAACCC TAAGATCCG, reverse 5'- GCAATGTGGCCTGACAG AAAGGGGAAATC; wildtype: forward 5'- CCTGGTTC GCAACCCTAAGATCC, reverse 5'- GCAATGTGGCCT GACAGAAAGGGGAAATC) to amplify a 202 bp amplicon. This also allowed confirmation of individual genotype.

\section{Statistics}

Association of genotype and gallbladder mucocele status was analyzed using the frequency procedure of SAS 9.2 (SAS Institute, Cary, NC), specifying Fisher's exact test and exact confidence intervals for the odds ratio.

\section{Results}

\section{Collection of affected and unaffected individuals}

Samples from 15 affected and 21 unaffected Shetland Sheepdogs were sequenced. Diagnosis of gallbladder mucocele was confirmed by ultrasound in 3 dogs, by sur- 
Table 1: Primers used for amplifying canine ABCB4.

\begin{tabular}{|c|c|c|c|}
\hline Exon & Forward Primer & Reverse Primer & Product Size \\
\hline 1 & TTC AGT TGG CTA TGA AAC ATT TGG & AGA CTA TCT TAA AGC ACT GAC TCC & 165 \\
\hline 2 & CCA AAA AAC ATA TAG TIT TGG GGA & GTC ATC TAG AAG TGC AAA CCA TTA AAC & 302 \\
\hline 3 & CCT AGT AAC ACC TAT TAA TAG TTC AGC C & СTC TGT AAG TTT GCA ATT ATT CTC & 202 \\
\hline 4 & CTT CCT GAA AGA GAT GAA TAA AGA AC & CAA AAG TAT GAC ATA AAT GAT ACA CTT AC & 225 \\
\hline 5 & GAA GAC CTC CTG CCT GTA ACC ACT & CAC ATG TGA AAA TGT TCC CGT TTC & 201 \\
\hline 6 & CAT GAA TGT TTC TTC TCT GTC CAG & GGT TCT TTG AAC CAG TGG AC & 143 \\
\hline 7 & GGC TAT GAT TAT GGA CTG TIT TCT TG & GGT TTC TTC ACG AAT ATT AGA AAG AC & 208 \\
\hline 8 & GCT TAT AAC TTC TTC TTG TGT TCT TIT G & GTG CAA GCC TCA AGG AAT TाT TाT TG & 143 \\
\hline 9 & CCT TAA AAG TGC AGT TGG TTG & GAA ATA AAA CCT GCC ACA GG & 249 \\
\hline 10 & CGT GAA GAG TGT TCT CTT TCT CTC & GCA GGG CTA ATT GGT AGC & 177 \\
\hline 11 & CTT GAT GCT TTA GAT GTC AGA TGG & CTC ACT TGC CTG AAG TCA AAG & 278 \\
\hline 12 & GAG ATA CAT CAG GAG CTC CTC C & CAG GTG TIT CGG GTT GAC TG & 189 \\
\hline 13 & GTA ACC CTG TTG CAT CAC AC & CTC AGC ATG GCA TTA GCT GC & 239 \\
\hline 14 & САA СТT АAC АТT ТTС ТСТ ТСТ TTC AG & GGA ATC ACT TGT GCC TGC & 256 \\
\hline 15 & CCA CTT TCT CCT GAT TCT CCT G & GGT GAA GCT GGC ATG AGA AC & 219 \\
\hline 16 & СTC TCT CTG GCT CTC ATG & CTC TAA TAG AAT GTG GAC TCG AG & 188 \\
\hline 17 & CTG ATG ATC AAA AGG GAC AAT C & GGA CTT CTC AAG TGC ACA C & 118 \\
\hline 18 & GAA GGT GTG TIT TGT GCC ACA G & ССС TाT CTG TCT CTC AAA TGG G & 141 \\
\hline 19 & CAT GGC TCC CTC TाT GCT TाT GC & СTC ACT GAA GCC TTC TIT GAC CCA C & 212 \\
\hline 20 & CGT TAT CCA GAA GTA AAA GCC C & CCT CAG GAA AGT ACT AGG GTC & 159 \\
\hline 21 & CCA GTC AAC TAC ACT AGA AGC TG & GAA CAA GTG AGT TाT TTC CAC CC & 260 \\
\hline 22 & GGT AAG CAC TAT GTC TIT GGA C & CAT TCA CCA GAC AGC AGA GAA C & 222 \\
\hline 23 & CAG ACC AAT TAT AAT AGC AAC ATT AAC & GCC TTA AAT AAG GTA CTA ACT TAA GC & 227 \\
\hline 24 & GAT ACC CAC ATG TCA CAA TGT TCC & TCC TGG TGC CAC TAC ATA GAC & 402 \\
\hline 25 & GTC CTA TAC CAA GTC ATG AGG AC & GGA AAC AGA GTG GAA AGA CC & 179 \\
\hline 26 & GGA ACT AAC TGT AGA CTA TAA TGC & GCT ATC TTA TCA ACA CCA AAT GG & 393 \\
\hline
\end{tabular}

gery in 5 dogs, and by histopathology in 7 dogs (Figure 1). Median age of Shetland Sheepdogs with a diagnosis of gallbladder mucocele was 9 years (range 5-12), which is similar to previous reports [13,15]. Ages for all the 21 unaffected Shetland Sheepdogs were not available, but the median age for those dogs whose ages were known (n = 12) was 9.5 years of age (range 5-14). Ages and breeds of the 3 affected non-Shetland Sheedogs are as follows: Cairn Terrier (11 years), Cocker Spaniel (13 years) and Pomeranian (11 years). Ages and breeds of the 20 unaffected non-Shetland Sheepdogs are indicated in Table 2.

\section{Sequencing of Canine $A B C B 4$}

Sequencing of all exons (1 to 26) of canine $A B C B 4$ was performed on genomic DNA from cheek swab samples (Shetland Sheepdogs) or from archived liver tissue (affected dogs that were not Shetland Sheepdogs). A single base pair insertion (G) was identified in exon 12 (Fig- ure 2) in 14 of 15 affected Shetland Sheepdogs, 1 of 21 unaffected Shetland Sheepdogs, and 3 affected dogs of other breeds (Cairn Terrier, Cocker Spaniel, and Pomeranian). The insertion mutation ( $A B C B 4$ 1583_1584G) is significantly associated $(\mathrm{P}<0.0001)$ with the diagnosis of gallbladder mucocele in Shetland Sheepdogs, with an odds ratio of 280 (95\% CI 12.7-12,350). In other dog breeds, $A B C B 4$ 1583_1584G is also significantly associated with the diagnosis of gallbladder mucocele $(\mathrm{P}<$ 0.0006). The frame shift generated by the insertion results in 4 premature stop codons within exon 12 . The full canine $A B C B 4$ gene contains 26 exons which encode essential structural elements that characterize $A B C$ transporters: two ATP binding domains and two substrate binding sites. Essential structural elements of ABCB4 normally contained within exon 12 and subsequent exons include both ATP binding sites and a substrate binding site. 
Table 2: Breed and age of unaffected dogs (non Shetland Sheepdogs).

\begin{tabular}{lcc}
\hline Breed & Number of Dogs & Age(years) \\
\hline Afghan Hound & 3 & $9.5 ; 10 ; 10$ \\
Asluki & 1 & 12 \\
Australian Shepherd & 1 & 10 \\
Brittany Spaniel & 1 & 11 \\
Corgi & 1 & 9 \\
English Cocker Spaniel & 1 & 12 \\
Golden Retriever & 1 & 9.5 \\
Jack Russell Terrier & 1 & 9 \\
Kelpie & 1 & 13 \\
Labrador Retriever & 3 & $9 ; 9.5 ; 9.5$ \\
Miniature Pinscher & 2 & $10 ; 13.5$ \\
Mixed Breed & 1 & 10 \\
Pitt Bull & 1 & 15 \\
Shih Tzu & 1 & 14 \\
Standard Poodle & 1 & 10 \\
\hline
\end{tabular}

A missense mutation in exon 15 of canine $A B C B 4$ was identified in the one affected Shetland Sheepdog that did not harbor $A B C B 4$ 1583_1584G. This SNP results in a nonhomologous amino acid substitution (alanine to serine) in exon 15 which may affect tertiary protein structure. However, this mutation was also present in 9 of the 21 unaffected Shetland Sheepdogs and 10 of the 15

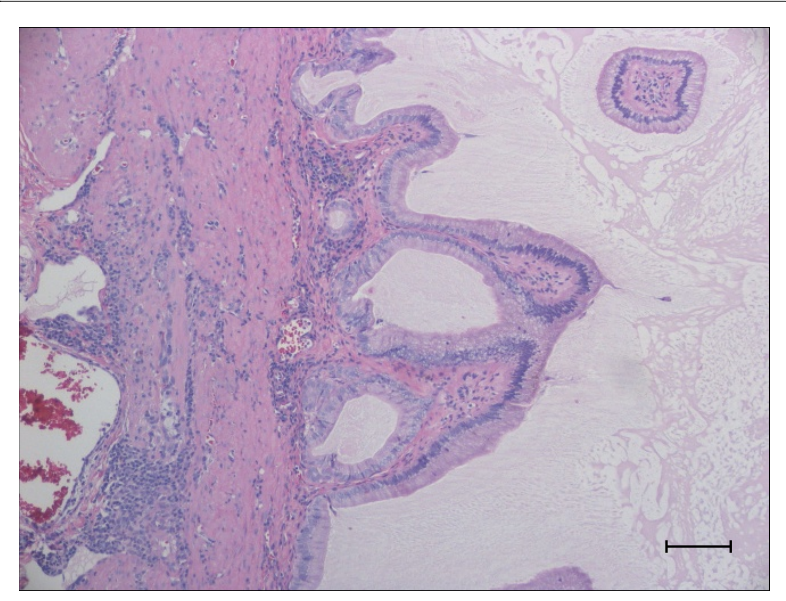

Figure 1 Gall Bladder. There is distention of the gall bladder with abundant luminal accumulations of mucus interspersed with scant amounts of bile. The mucosa of the gall bladder is lined by moderately hyperplastic columnar epithelial cells with accentuation of the normal folds by accumulations of mucus. Within the lamina propria and the tunica muscularis there are occasional multifocal to perivascular accumulations of lymphocytes and rare plasma cells. Hematoxylin and eosin staining. Bar $=250 \mu \mathrm{m}$.

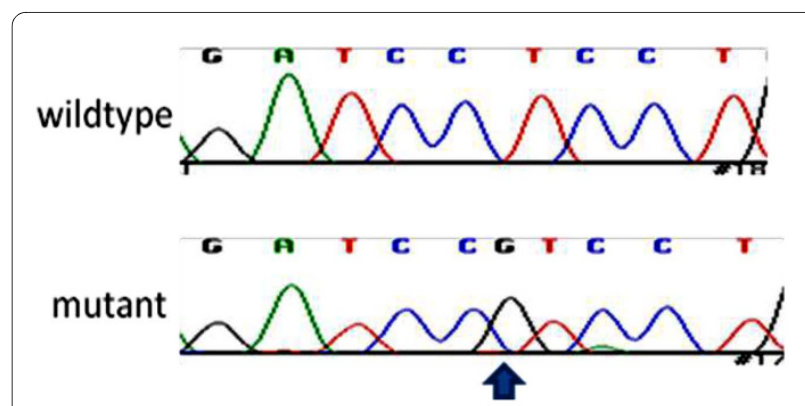

Figure 2 Electropherograms for wildtype and mutant canine $A B C B 4$. The insertion is indicated by an arrow.

affected Shetland Sheepdogs, so its significance is unclear. No obvious differences were apparent in disease severity or biochemical parameters in the affected dogs with the mutation in exon 15 .

\section{Confirmation of Insertion by Allele Specific PCR}

To confirm the presence of $A B C B 4$ 1583_1584G as well as determine the genotype of each dog, allele specific primers were designed and used to amplify the region of interest in exon 12 (Figure 3). All dogs harboring the insertion were heterozygous at the mutant allele suggesting a dominant mode of inheritance with incomplete penetrance. None of the dogs in the study were homozygous for the mutant allele. Genotype frequencies are shown in Table 3.

\section{Discussion}

Over three dozen disease-causing mutations in human ABCB4 have been described $[5,7,9,10]$. The disease spectrum ranges from severe (debilitating diseases of young children that require liver transplantation) to mild. Disease severity often depends on the nature of the mutation. Milder disease occurs when the ABCB4 gene mutation reduces but does not eliminate transport activity of the protein. Similarly, milder forms of disease exist in patients that are heterozygous for mutations that eliminate transporter activity (i.e., truncations).

The canine $A B C B 4$ insertion mutation reported here results in a truncation that eliminates more than $50 \%$ of the protein. This mutation was significantly associated with the diagnosis of gallbladder mucocele in Shetland Sheepdogs as well as other dog breeds. The etiology of gallbladder mucoceles in dogs is currently unknown, but extrahepatic bile duct obstruction is not a common component of the disease (as has been reported in people with gallbladder mucoceles) [18]. The results reported here provide evidence that dysfunction of $A B C B 4$ is likely involved. Hepatocyte PC transport, and therefore bile PC content, in dogs that harbor ABCB4 1583_1584G would be decreased compared to wildtype dogs. Biliary epithelial lining cells would be subjected to bile salt-induced injury because of diminished ability to form mixed 
Table 3: $A B C B 4$ genotype frequencies in gallbladder mucocele affected and unaffected animals.

\begin{tabular}{lcc}
\hline & Shetland Sheepdog (affected) & Shetland Sheepdog (unaffected) \\
\hline$A B C B 4$ 1583_1584G (wildtype) & 1 & 14 \\
$A B C B 4$ 1583_1584G (heterozygous) & 0 \\
$A B C B 4$ 1583_1584G (homozygous) & Other breeds (affected) & 0 \\
& 0 & Other breeds (unaffected) \\
$A B C B 4$ 1583_1584G (wildtype) & 3 & 0 \\
$A B C B 4$ 1583_1584G (heterozygous) & 0 \\
$A B C B 4$ 1583_1584G (homozygous) & 0 \\
\hline
\end{tabular}

micelles [19]. A universal physiologic response of epithelial linings to injury is mucinous hyperplasia, a histopathologic finding frequently described in dogs diagnosed with gallbladder mucocele. Furthermore, exposure to bile salts has been shown to stimulate mucin secretion in cultured canine gallbladder epithelial cells [20]. Thus, gallbladder epithelium in dogs that harbor $A B C B 4$ 1583_1584G undergoes greater exposure to unneutralized bile salts than that of wildtype dogs, resulting in greater mucin secretion, mucinous hyperplasia, and eventually mucocele formation.

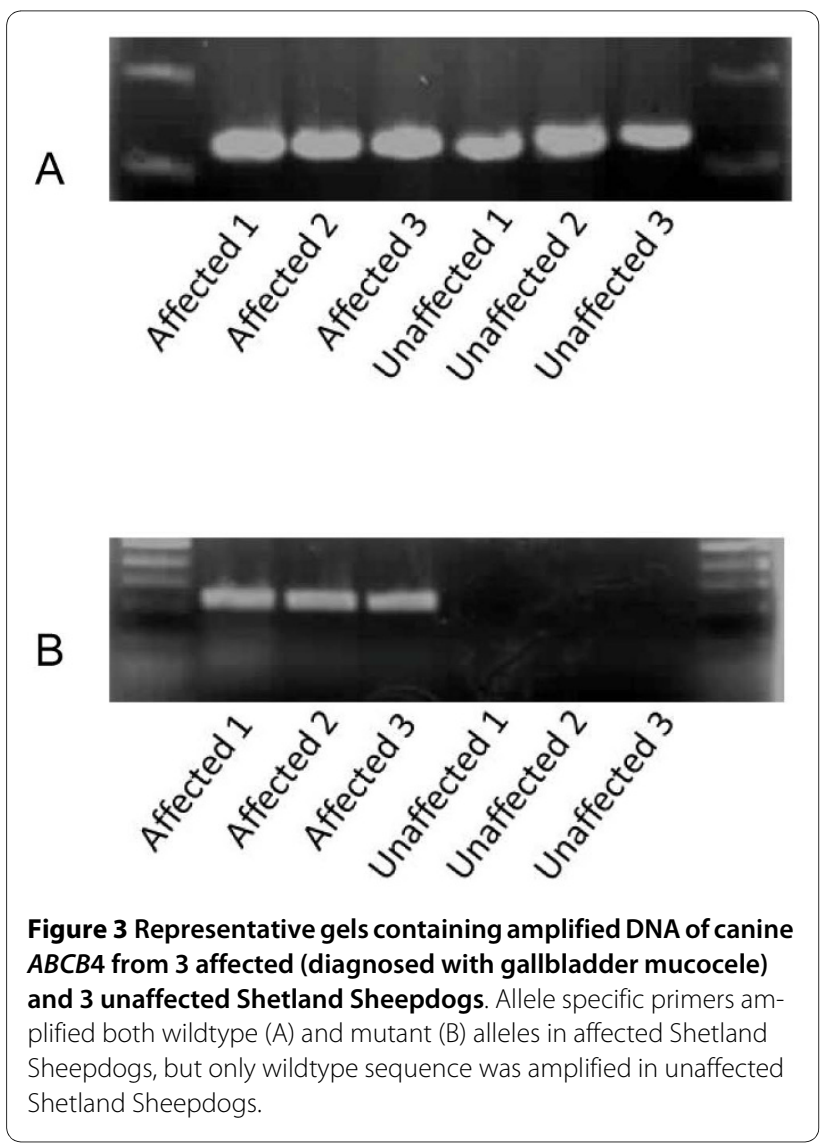

Because gallbladder mucoceles are a relatively new disease condition in dogs, a "gold standard" diagnosis has not yet been defined. Inclusion criteria used in previous publications consist of surgical or necropsy diagnosis (macroscopic appearance), ultrasonographic diagnosis, and/or histopathological diagnosis (microscopic appearance) $[14,15,21]$. Each of these criteria has limitations for diagnosing gallbladder mucoceles. A number of ultrasonographic findings have been associated with gallbladder mucocele, and there is sometimes disagreement among ultrasonographers as to what constitutes a gallbladder mucocele. Additional confusion is created by terminology such as "early" or "developing" gallbladder mucocole. Because of the gallbladder's universal physiological response to irritation (e.g., mucus secretion), some might argue that even a histopathological diagnosis of gallbladder mucocele may generate some speculation. It seems reasonable, therefore, to entertain the possibility that our study population ("affecteds") might contain false positives and that our control population ("unaffecteds") might contain false negatives despite the fact that currently acceptable criteria were used to identify these populations. However, the statistical difference between groups was so dramatic (based on current criteria) that statistical relevance would still hold even if some errors exist in the study or control population based on diagnostic criteria that may be defined in the future. The association of $A B C B 4$ 1583_1584G with gallbladder mucoceles in dogs represents an important advancement in our understanding of the disease.

A number of other potential etiologies have been suggested for gallbladder mucoceles in dogs. These include primary or secondary motility disorders of gallbladder motility, a secondary complication of dyslipidemias (Shetland Sheepdogs and Miniature Schnauzers) in particular, and primary disorders of mucus-secreting cells [13]. Recently, hyperadrenocorticism was reported to be significantly associated with the diagnosis of gallbladder mucocele in dogs [21]. Our findings do not rule out other 
potential etiologies, and it is certainly possible that $A B C B 4$ 1583_1584G could be one of many contributing factors to gallbladder mucoceles in dogs.

Many of the dogs from our study and other studies were severely affected at the time of diagnosis with some dogs dying of their disease despite surgical intervention $[13,15]$. Our discovery of the insertion mutation in canine $A B C B 4$ allows early identification of dogs predisposed to gallbladder mucocele formation. This creates a number of beneficial applications for dogs. Genotyping of young dogs for $A B C B 4$ 1583_1584G would allow veterinarians to closely monitor for development of a gallbladder mucocele in affected dogs. Surgical intervention could be performed earlier in the disease process before diseaseinduced morbidity places the patient at higher risk for intra- and post-operative complications.

Another benefit of genotyping dogs for the $A B C B 4$ 1583 1584G is the possibility of medical or dietary management to prevent or at least delay the onset of mucocele formation. Currently, no medical treatment options have been systematically evaluated for managing dogs with gallbladder mucoceles primarily because information regarding the etiology of the disease has been lacking. However, ursodeoxycholic acid has been suggested [22]. Some human patients with ABCB4-associated biliary disease benefit from treatment with ursodeoxycholic acid, a relatively hydrophilic and much less cytotoxic bile acid than most endogenous bile salts [4]. Studies to determine bile composition in wildtype dogs and dogs with the ABCB4 1583_1584G mutation should be performed in order to further characterize the disease. One would expect affected dogs to have bile with lower phospholipid concentrations than wildtype dogs, and thus a greater proportion of simple micelles rather than mixed micelles. These studies would also be important to determine how useful affected dogs would be as a model for the various biliary diseases in people that result from similar ABCB4 mutations.

The authors speculate that occurrence of gallbladder mucoceles in dogs is inherited in a dominant fashion with incomplete penetrance, however further research is required to confirm the mode of inheritance. While it is possible that the one unaffected carrier of the $A B C B 4$ 1583_1584G insertion may develop biliary disease in the future, there was no evidence of disease at 9 years of age. No dogs in this study population were homozygous for the mutation. Because a more severe phenotype is observed in people homozygous for mutations resulting in elimination of $\mathrm{ABCB} 4$ protein function, one would speculate that the same would be true for dogs. In people with PFIC (type 3), the disease manifests during early childhood and is fatal without a liver transplant [4]. It is possible that homozygosity for the mutation results in death of affected dogs either during embryonic development or in early puppyhood.

In conclusion, the $A B C B 41583 \_1584 G$ is strongly associated with the diagnosis of gallbladder mucocele in dogs. Results of this study provide the first spontaneous animal model for studying a number of potentially lethal or severely debilitating hepatobiliary diseases in people that are also associated with ABCB4 dysfunction. This canine model may be useful for studying potential medical and/ or dietary treatments for ABCB4-associated hepatobiliary diseases in people.

\section{List of abbreviations}

$\mathrm{ABC}$ : adenosine triphosphate-binding cassette; $\mathrm{ABCB} 4$ : adenosine triphosphate-binding cassette, subfamily $B$, member 4; PC: Phosphatidylcholine, G: guanine.

\section{Competing interests}

The authors declare that a patent application has been filed by Washington State University listing two of the authors as inventors (KLM, JDM).

\section{Authors' contributions}

JDM performed experiments; JSM and KRS assisted in acquiring and interpreting data; SNW performed statistical analysis; KLM conceived and designed the research project. All authors made critical revision of the manuscript for important intellectual content. All authors read and approved the final manuscript.

\section{Acknowledgements}

The authors would like to thank Mary B. Mahaffey, DVM for promoting sample submission within the American Shetland Sheepdog Association. The authors would also like to thank all dog owners for donating samples and sharing data from their dogs' medical records. This work was supported by a Washington State University College of Veterinary Medicine Intramural Grant and Proceeds from the Veterinary Clinical Pharmacology Laboratory at Washington State University.

\section{Author Details}

'Department of Veterinary Clinical Sciences, College of Veterinary Medicine, Washington State University, Pullman, WA 99164-6610, USA, 2USDA-ARS Animal Disease Research Unit, Pullman, WA 99164-6630, USA, ${ }^{3}$ Department of Veterinary Microbiology \& Pathology, College of Veterinary Medicine, Washington State University, Pullman, WA 99164, USA and ${ }^{4}$ Center for Integrated Biotechnology, Washington State University, Pullman, WA, 99164 , USA

Received: 20 November 2009 Accepted: 3 July 2010 Published: 3 July 2010

\section{References}

1. Pellicoro A, Faber KN: Review article: The function and regulation of proteins involved in bile salt biosynthesis and transport. Aliment Pharmacol Ther 2007, 26:149-160.

2. Elferink RO, Groen AK: Genetic defects in hepatobiliary transport. Biochim Biophys Acta 2002, 1586:129-145.

3. Coleman R, lqbal S, Godfrey PP, Billington D: Membranes and bile formation. Composition of several mammalian biles and their membrane-damaging properties. Biochem J 1979, 178:201-208.

4. Oude Elferink RP, Paulusma CC: Function and pathophysiological importance of ABCB4 (MDR3 P-glycoprotein). Pflugers Arch 2007, 453:601-610.

5. Davit-Spraul A, Gonzales E, Baussan C, Jacquemin E: Progressive familia intrahepatic cholestasis. Orphanet J Rare Dis 2009, 4:1.

6. Trauner M, Fickert $P$, Wagner M: MDR3 (ABCB4) defects: a paradigm for the genetics of adult cholestatic syndromes. Semin Liver Dis 2007, 27:77-98. 
7. Dean $M$, Annilo T: Evolution of the ATP-binding cassette (ABC) transporter superfamily in vertebrates. Annu Rev Genomics Hum Genet 2005, 6:123-142.

8. Delaunay JL, Durand-Schneider AM, Delautier D, Rada A, Gautherot J, Jacquemin E, Ait-Slimane T, Maurice M: A missense mutation in ABCB4 gene involved in progressive familial intrahepatic cholestasis type 3 leads to a folding defect that can be rescued by low temperature. Hepatology 2009, 49:1218-1227.

9. Gonzales E, Davit-Spraul A, Baussan C, Buffet C, Maurice M, Jacquemin E: Liver diseases related to MDR3 (ABCB4) gene deficiency. Front Biosci 2009, 14:4242-4256

10. Nakken KE, Labori KJ, Rodningen OK, Nakken S, Berge KE, Eiklid K, Raeder $M G: A B C B 4$ sequence variations in young adults with cholesterol gallstone disease. Liver Int 2009, 29:743-747.

11. Smit JJ, Schinkel AH, Oude Elferink RP, Groen AK, Wagenaar E, van Deemter $L$, Mol CA, Ottenhoff R, van der Lugt NM, van Roon MA, van der Valkc MA, Offerhausd GJA, Bernsc AJM, Borst P: Homozygous disruption of the murine mdr2 P-glycoprotein gene leads to a complete absence of phospholipid from bile and to liver disease. Cell 1993, 75:451-462.

12. Baghdasaryan A, Fickert P, Fuchsbichler A, Silbert D, Gumhold J, Horl G, Langner C, Moustafa T, Halilbasic E, Claudel T, Trauner M: Role of hepatic phospholipids in development of liver injury in Mdr2 (Abcb4) knockout mice. Liver Int 2008:948-958.

13. Aguirre AL, Center SA, Randolph JF, Yeager AE, Keegan AM, Harvey HJ, Erb HN: Gallbladder disease in Shetland Sheepdogs: 38 cases (1995-2005). J Am Vet Med Assoc 2007, 231:79-88.

14. Besso JG, Wrigley RH, Gliatto JM, Webster CR: Ultrasonographic appearance and clinical findings in 14 dogs with gallbladder mucocele. Vet Radiol Ultrasound 2000, 41:261-271.

15. Pike FS, Berg J, King NW, Penninck DG, Webster CR: Gallbladder mucocele in dogs: 30 cases (2000-2002). J Am Vet Med Assoc 2004, 224:1615-1622.

16. Worley DR, Hottinger HA, Lawrence HJ: Surgical management of gallbladder mucoceles in dogs: 22 cases (1999-2003). J Am Vet Med Assoc 2004, 225:1418-1422.

17. Newell SM, Selcer BA, Mahaffey MB, Gray ML, Jameson PH, Cornelius LM, Downs MO: Gallbladder mucocele causing biliary obstruction in two dogs: ultrasonographic, scintigraphic, and pathological findings. J Am Anim Hosp Assoc 1995, 31:467-472.

18. Nahrwold D: Textbook of Surgery: The Biological Basis of Modern Surgical Practice Philadelphia: W. B. Saunders; 1991.

19. Anwer MS, Meyer DJ: Bile acids in the diagnosis, pathology, and therapy of hepatobiliary diseases. Vet Clin North Am Small Anim Pract 1995 25:503-517.

20. Klinkspoor JH, Yoshida T, Lee SP: Bile salts stimulate mucin secretion by cultured dog gallbladder epithelial cells independent of their detergent effect. Biochem J 1998, 332:257-262.

21. Mesich ML, Mayhew PD, Paek M, Holt DE, Brown DC: Gall bladder mucoceles and their association with endocrinopathies in dogs: a retrospective case-control study. J Small Anim Pract 2009, 50:630-635.

22. Walter R, Dunn ME, d'Anjou MA, Lecuyer M: Nonsurgical resolution of gallbladder mucocele in two dogs. J Am Vet Med Assoc 2008, 232:1688-1693.

\section{doi: 10.1186/1476-5926-9-6}

Cite this article as: Mealey et al., An insertion mutation in ABCB4 is associated with gallbladder mucocele formation in dogs Comparative Hepatology 2010, 9:6

\section{Submit your next manuscript to BioMed Central} and take full advantage of:

- Convenient online submission

- Thorough peer review

- No space constraints or color figure charges

- Immediate publication on acceptance

- Inclusion in PubMed, CAS, Scopus and Google Scholar

- Research which is freely available for redistribution

Submit your manuscript at www.biomedcentral.com/submit
C) Biomed Central 